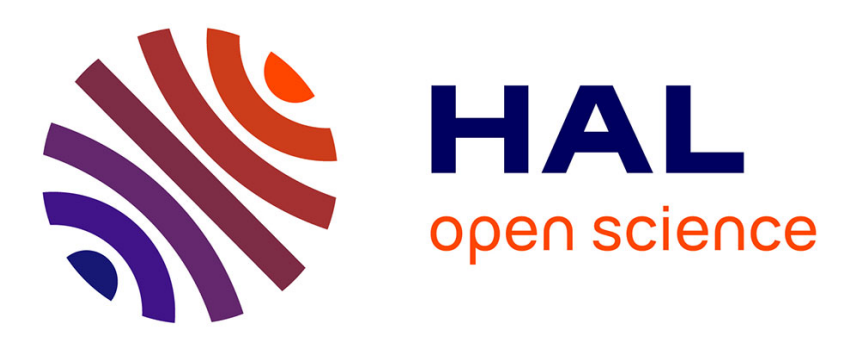

\title{
On a conjecture concerning some automatic continuity theorems
}

\author{
Mohammed El Azhari
}

\section{To cite this version:}

Mohammed El Azhari. On a conjecture concerning some automatic continuity theorems. Rendiconti del Circolo Matematico di Palermo, 2012, 61 (1), pp.13-17. 10.1007/s12215-011-0070-2 . hal01303274

\section{HAL Id: hal-01303274 \\ https://hal.science/hal-01303274}

Submitted on 17 Apr 2016

HAL is a multi-disciplinary open access archive for the deposit and dissemination of scientific research documents, whether they are published or not. The documents may come from teaching and research institutions in France or abroad, or from public or private research centers.
L'archive ouverte pluridisciplinaire HAL, est destinée au dépôt et à la diffusion de documents scientifiques de niveau recherche, publiés ou non, émanant des établissements d'enseignement et de recherche français ou étrangers, des laboratoires publics ou privés. 


\title{
ON A CONJECTURE CONCERNING SOME AUTOMATIC CONTINUITY THEOREMS
}

\author{
M.El Azhari
}

ABSTRACT. Let A and B be commutative locally convex algebras with unit. A is assumed to be a uniform topological algebra. Let $\Phi$ be an injective homomorphism from A to B. Under additional assumptions, we characterize the continuity of the homomorphism $\Phi^{-1} / \operatorname{Im} \Phi$ by the fact that the radical (or strong radical) of the closure of $\operatorname{Im} \Phi$ has only zero as a common point with $\operatorname{Im} \Phi$. This gives an answer to a conjecture concerning some automatic continuity theorems on uniform topological algebras.

1. INTRODUCTION. Let A and B be commutative locally convex algebras with unit. A is assumed to be a uniform topological algebra. Let $\Phi$ be an injective homomorphism from A to B. Under which conditions is $\Phi^{-1} / \operatorname{Im} \Phi$ continuous?

Under additional assumptions such as:

(1) A is weakly regular and functionally continuous, B an $1 \mathrm{mc}$ algebra, and $(\operatorname{Im} \Phi)^{-}$(the closure of $\operatorname{Im} \Phi)$ is a semisimple Q-algebra; or

(2) A is weakly $\sigma^{*}$-compact-regular, and $(\operatorname{Im} \Phi)^{-}$is a strongly semisimple Q-algebra;

it is shown in [5] that $\Phi^{-1} / \operatorname{Im} \Phi$ is continuous, which improves earlier results by Bedaa, Bhatt and Oudadess ([2]).

The following examples show that the hypothesis $(\operatorname{Im} \Phi)^{-}$is a Q-algebra in (1) and (2) cannot be omitted.

Example 1. Let $A=C[0,1]$ be the algebra of all complex continuous functions on the closed unit interval $[0,1]$. A is a uniform Banach algebra under the supnorm. Since $\mathrm{M}(\mathrm{A})$ is homeomorphic to $[0,1]$, it follows that $\mathrm{A}$ is weakly regular. Consider $\mathrm{B}=\mathrm{C}[0,1]$. For any countable compact subset $\mathrm{K}$ of $[0,1]$, and $f \in B$, we put $p_{K}(f)=\sup \{|f(x)|, x \in K\}$. B is a complete uT-algebra under the system $\left(\mathrm{p}_{\mathrm{K}}\right)_{\mathrm{K}}$. Consider $\Phi: \mathrm{A} \rightarrow \mathrm{B}, \Phi(\mathrm{f})=\mathrm{f}$. Then $(\operatorname{Im} \Phi)^{-}=\mathrm{B}$ is semisimple but not a Q-algebra. Clearly $\Phi^{-1} / \operatorname{Im} \Phi$ is not continuous.

Example 2. Let $A=C_{b}(R)$ be the algebra of all complex continuous bounded functions on the real line. A is a uniform Banach algebra under the supnorm. A is weakly $\sigma^{*}$-compact-regular [2, Remark (4)]. Let $B=C(R)$ be the algebra of all complex continuous functions on $R$, with the compact-open topology. Consider $\Phi: A \rightarrow B, \Phi(f)=f$. Then $(\operatorname{Im} \Phi)^{-}=\mathrm{C}(\mathrm{R})$ is strongly semisimple but not a Q-algebra. Clearly $\Phi^{-1} / \operatorname{Im} \Phi$ is not continuous.

In [2], the authors conjectured that the semisimplicity of $(\operatorname{Im} \Phi)^{-}$in (1) (and strong semisimplicity of $(\operatorname{Im} \Phi)^{-}$in (2)) can be omitted. According to the proofs in [2] and [5], the 
semisimplicity of $(\operatorname{Im} \Phi)^{-}$in (1) can be replaced by $\operatorname{Im} \Phi \cap \mathrm{R}\left((\operatorname{Im} \Phi)^{-}\right)=\{0\}$, and the strong semisimplicity of $(\operatorname{Im} \Phi)^{-}$in $(2)$ can be replaced by $\operatorname{Im} \Phi \cap \operatorname{SR}\left((\operatorname{Im} \Phi)^{-}\right)=\{0\}$.

In this paper, we show that if $\mathrm{A}$ is weakly regular and functionally continuous, $\mathrm{B}$ an $1 \mathrm{mc}$ algebra, and $(\operatorname{Im} \Phi)^{-}$is a Q-algebra, then the continuity of $\Phi^{-1} / \operatorname{Im} \Phi$ is equivalent to $\operatorname{Im} \Phi \cap \operatorname{R}\left((\operatorname{Im} \Phi)^{-}=\{0\}\right.$. We also show that if $\mathrm{A}$ is weakly $\sigma^{*}$-compact-regular, B has continuous product, and $(\operatorname{Im} \Phi)^{-}$is a Q-algebra, then the continuity of $\Phi^{-1} / \operatorname{Im} \Phi$ is equivalent to $\operatorname{Im} \Phi \cap \operatorname{SR}\left((\operatorname{Im} \Phi)^{-}=\{0\}\right.$.

2. PRELIMINARIES. All algebras considered are over the field $\mathbb{C}$, commutative, and having a unit element. A topological algebra is an algebra which is also a Hausdorff topological vector space such that the multiplication is separately continuous. A locally convex algebra (lc algebra) is a topological algebra whose topology is locally convex. A locally multiplicatively convex algebra (lmc algebra) is a topological algebra whose topology is determined by a family of submultiplicative seminorms. A uniform seminorm on an algebra $A$ is a seminorm $p$ such that $\mathrm{p}\left(\mathrm{x}^{2}\right)=\mathrm{p}(\mathrm{x})^{2}$ for all $\mathrm{x} \in \mathrm{A}$. Such a seminorm is submultiplicative [4]. A uniform topological algebra (uT-algebra) is a topological algebra whose topology is determined by a family of uniform seminorms. A uniform normed algebra is a normed algebra $(\mathrm{A},\|\|)$ such that $\left\|\mathrm{x}^{2}\right\|=\|\mathrm{x}\|^{2}$ for all $x \in A$. Let $A$ be an algebra and $x \in A$, we denote by $\operatorname{sp}_{A}(x)$ the spectrum of $x$ and $r_{A}(x)$ the spectral radius of $\mathrm{x}$. For an algebra $\mathrm{A}, \mathrm{M}^{*}(\mathrm{~A})$ denotes the set of all nonzero multiplicative linear functionals on A. For a topological algebra A, M(A) denotes the set of all nonzero continuous multiplicative linear functionals on $\mathrm{A}$. A topological algebra $\mathrm{A}$ is functionally continuous if $\mathrm{M}^{*}(\mathrm{~A})=\mathrm{M}(\mathrm{A})$. A topological algebra is a Q-algebra [7] if the set of invertible elements is open. A topological algebra is weakly regular [2] if given a closed subset $F$ of $M(A), F \neq M(A)$, there exists a nonzero $x \in A$ such that $f(x)=0$ for all $f \in F$. A topological algebra $A$ is weakly $\sigma^{*}$-compact-regular [2] if given a compact subset $K$ of $M^{*}(A), K \neq M^{*}(A)$, there exists a nonzero $x \in A$ such that $f(x)=0$ for all $f \in K$. We use $R(A)$ to denote the radical of an algebra A. If $R(A)=\{0\}$, we say that $A$ is semisimple. Let $A$ be a topological algebra with $M(A) \neq \varnothing$, the set $\{x \in A, f(x)=0$ for all $f \in M(A)\}$ is called the strong radical of $A$ and denoted by $S R(A)$. If $\mathrm{SR}(\mathrm{A})=\{0\}$, we say that $\mathrm{A}$ is strongly semisimple. Let $\mathrm{A}$ be an lmc algebra, if $\mathrm{A}$ is complete or a Q-algebra, then $\mathrm{R}(\mathrm{A})=\mathrm{SR}(\mathrm{A})$.

\section{RESULTS}

Theorem 3.1. Let $\mathrm{A}$ be a weakly regular, functionally continuous, $\mathrm{uT}$-algebra. Let $\mathrm{B}$ be an $\operatorname{lmc}$ algebra, and let $\Phi: \mathrm{A} \rightarrow \mathrm{B}$ be a one-to-one homomorphism such that $(\operatorname{Im} \Phi)^{-}$is a $\mathrm{Q}$-algebra. Then the following are equivalent:

(1) $\Phi^{-1 /} \operatorname{Im} \Phi$ is continuous.

(2) $\operatorname{Im} \Phi$ is functionally continuous.

(3) $\Phi^{*}: \mathrm{M}\left((\operatorname{Im} \Phi)^{-}\right) \rightarrow \mathrm{M}(\mathrm{A}), \Phi^{*}(\mathrm{f})=$ fo $\Phi$, is surjective.

(4) $\operatorname{Im} \Phi \cap \mathrm{R}\left((\operatorname{Im} \Phi)^{-}\right)=\{0\}$.

Proof: $(1)=>(2)$ : Let $F \in M^{*}(\operatorname{Im} \Phi), F=F o \Phi o\left(\Phi^{-1} / \operatorname{Im} \Phi\right)$ is continuous since Fo $\Phi$ and $\Phi^{-1 /} \operatorname{Im} \Phi$ are continuous. 
$(2)=>(3)$ : Let $f \in M(A)$ and $F=f o\left(\Phi^{-1} / \operatorname{Im} \Phi\right), F \in M^{*}(\operatorname{Im} \Phi)=M(\operatorname{Im} \Phi)$ and $f=F o \Phi . F$ can be extended to an $\mathrm{F}^{-} \in \mathrm{M}\left((\operatorname{Im} \Phi)^{-}\right)$. We have $\mathrm{f}=\mathrm{F}^{-} \mathrm{o} \Phi$. This shows that $\Phi^{*}$ is surjective. $(3)=>(1)$ : By [5, Theorem 2.1], the topology of $A$ is defined by a family $\left\{p_{S}, s \in S\right\}$ of submultiplicative seminorms such that (i ) for all $x \in A$ and $s \in S$ with $p_{s}(x)=1$, there exists $f \in M(A)$ such that $|f(x)|=1$. Let $s \in S$ and $y \in \operatorname{Im} \Phi$ with $p_{S}\left(\Phi^{-1}(y)\right) \neq 0$. By (i ), there exists $\mathrm{f} \in \mathrm{M}(\mathrm{A})$ such that $\left|\mathrm{f}\left(\Phi^{-1}(\mathrm{y})\right)\right|=\mathrm{p}_{\mathrm{S}}\left(\Phi^{-1}(\mathrm{y})\right)$. Since $\Phi^{*}$ is surjective, there exists $\mathrm{F} \in \mathrm{M}\left((\operatorname{Im} \Phi)^{-}\right)$ such that $\mathrm{f}=$ Fo $\Phi$. We have $\mathrm{p}_{\mathrm{S}}\left(\Phi^{-1}(\mathrm{y})\right)=\left|\mathrm{f}\left(\Phi^{-1}(\mathrm{y})\right)\right|=|\mathrm{F}(\mathrm{y})| \leq \mathrm{r}_{\mathrm{C}}(\mathrm{y})$, where $\mathrm{C}=(\mathrm{Im} \Phi)^{-}$. Since $C$ is a Q-algebra, $r_{C}$ is continuous at 0 [7, Proposition 13.5]. Then $\Phi^{-1} / \operatorname{Im} \Phi$ is continuous. (3) $=>(4)$ : Let $\mathrm{y} \in \operatorname{Im} \Phi \cap \mathrm{R}\left((\operatorname{Im} \Phi)^{-}\right)$, there exists $\mathrm{x} \in \mathrm{A}$ such that $\mathrm{y}=\Phi(\mathrm{x})$ and $\mathrm{F}(\Phi(\mathrm{x}))=0$ for all $F \in M\left((\operatorname{Im} \Phi)^{-}\right)$. Then $f(x)=0$ for all $f \in M(A)$ since $\Phi^{*}$ is surjective. Hence $x=0$ and so $\mathrm{y}=\Phi(\mathrm{x})=0$ since $\mathrm{A}$ is a uT-algebra.

(4) $=>(3): \Phi^{*}$ is well defined and continuous. Since $(\operatorname{Im} \Phi)^{-}$is a Q-algebra, $\mathrm{M}\left((\operatorname{Im} \Phi)^{-}\right)$is compact [6, p.187 ], thus $\Phi^{*}\left(\mathrm{M}\left((\operatorname{Im} \Phi)^{-}\right)\right.$is compact. Suppose that $\Phi^{*}$ is not surjective. By the weak regularity of $A$, there exists a nonzero $x \in A$ such that $f(\Phi(x))=0$ for all $f \in M\left((\operatorname{Im} \Phi)^{-}\right)$. Since $(\operatorname{Im} \Phi)^{-}$is a Q-algebra, it follows that $\Phi(\mathrm{x}) \in \operatorname{Im} \Phi \cap \mathrm{R}\left((\operatorname{Im} \Phi)^{-}\right)=\{0\}$, and then $\mathrm{x}=0$, a contradiction.

Theorem 3.2. Let A be a weakly $\sigma^{*}$-compact-regular, uT-algebra. Let B be an lc algebra with continuous product, and $\Phi: \mathrm{A} \rightarrow \mathrm{B}$ be a one-to-one homomorphism such that $(\operatorname{Im} \Phi)^{-}$is a Q-algebra. The following are equivalent:

(1) $\Phi^{-1 /} \operatorname{Im} \Phi$ is continuous.

(2) $\operatorname{Im} \Phi \cap \operatorname{SR}\left((\operatorname{Im} \Phi)^{-}\right)=\{0\}$.

(3) $\Phi^{* *}: \mathrm{M}\left((\operatorname{Im} \Phi)^{-}\right) \rightarrow \mathrm{M}^{*}(\mathrm{~A}), \Phi^{* *}(\mathrm{f})=$ fo $\Phi$, is surjective.

Proof: $(1)=>(2)$ : The topology of $A$ is determined by a family $\left\{p_{u}, u \in U\right\}$ of uniform seminorms. For each $u \in U$, let $N_{u}=\left\{x \in A, p_{u}(x)=0\right\}$ and $A_{u}$ be the Banach algebra obtained by completing $A / N_{u}$ in the norm $\left\|x_{u}\right\|_{u}=p_{u}(x), x_{u}=x+N_{u}$. It is clear that $A_{u}$ is a uniform Banach algebra. For each $u \in U$, let $M_{u}(A)=\left\{f \in M(A),|f(x)| \leq p_{u}(x)\right.$ for all $\left.x \in A\right\}$. Let $u \in U$ and $x \in A$, $\mathrm{p}_{\mathrm{u}}(\mathrm{x})=\left\|\mathrm{x}_{\mathrm{u}}\right\|_{\mathrm{u}}=\mathrm{r}_{\mathrm{u}}\left(\mathrm{x}_{\mathrm{u}}\right)=\sup \left\{\left|\mathrm{g}\left(\mathrm{x}_{\mathrm{u}}\right)\right|, \mathrm{g} \in \mathrm{M}\left(\mathrm{A}_{\mathrm{u}}\right)\right\}=\sup \left\{|\mathrm{f}(\mathrm{x})|, \mathrm{f} \in \mathrm{M}_{\mathrm{u}}(\mathrm{A})\right\}$ by [7, Proposition 7.5 ] $\left(r_{u}\right.$ is the spectral radius on $\left.A_{u}\right)$. Let $u \in U$ and $y \in \operatorname{Im} \Phi, p_{u}\left(\Phi^{-1}(y)\right)=\sup \left\{\mid\right.$ foo $\left.\left.\Phi^{-1}(y)\right) \mid, f \in M_{u}(A)\right\}$. Let $f \in M_{u}(A)$, fo $\Phi^{-1} \in M(\operatorname{Im} \Phi)=M\left((\operatorname{Im} \Phi)^{-}\right)$since $\Phi^{-1} / \operatorname{Im} \Phi$ is continuous and $B$ has continuous product. Then $\mathrm{p}_{\mathrm{u}}\left(\Phi^{-1}(\mathrm{y})\right) \leq \sup \left\{|\mathrm{F}(\mathrm{y})|, \mathrm{F} \in \mathrm{M}\left((\operatorname{Im} \Phi)^{-}\right)\right\}$for all $\mathrm{u} \in \mathrm{U}$ and $\mathrm{y} \in \operatorname{Im} \Phi$. Let $\mathrm{y} \in \operatorname{Im} \Phi \cap \operatorname{SR}\left((\operatorname{Im} \Phi)^{-}\right)$, we have $\mathrm{p}_{\mathrm{u}}\left(\Phi^{-1}(\mathrm{y})\right)=0$ for all $\mathrm{u} \in \mathrm{U}$, then $\Phi^{-1}(\mathrm{y})=0$ and so $\mathrm{y}=0$. $(2)=>(3)$ : $\Phi^{* *}$ is continuous. Since $(\operatorname{Im} \Phi)^{-}$is a Q-algebra, $\mathrm{M}\left((\operatorname{Im} \Phi)^{-}\right)$is compact [6, p.187], thus $\Phi^{* *}\left(\mathrm{M}\left((\operatorname{Im} \Phi)^{-}\right)\right)$is compact. Suppose that $\Phi^{* *}$ is not surjective. Since A is $\sigma^{*}$-compactregular, there exists a nonzero $\mathrm{x} \in \mathrm{A}$ such that $\mathrm{f}(\Phi(\mathrm{x}))=0$ for all $\mathrm{f} \in \mathrm{M}\left((\operatorname{Im} \Phi)^{-}\right)$. This gives $\Phi(\mathrm{x}) \in \operatorname{Im} \Phi \cap \operatorname{SR}\left((\operatorname{Im} \Phi)^{-}\right)=\{0\}$, and then $\mathrm{x}=0$, a contradiction.

(3) $=>$ (1): Similar to the proof of (3) $=>$ (1) in Theorem 3.1.

Here is an example such that A is weakly regular, uniform Banach algebra, B is a Banach algebra, $\Phi: \mathrm{A} \rightarrow \mathrm{B}$ is a one-to-one homomorphism, and $\operatorname{Im} \Phi \cap \mathrm{R}\left((\operatorname{Im} \Phi)^{-}\right)=\{0\}$ but $\mathrm{R}\left((\operatorname{Im} \Phi)^{-}\right) \neq\{0\}$. 
Example. Let $\mathrm{A}=\mathrm{C}[0,1]$ be the algebra of all complex continuous functions on the closed unit interval $[0,1]$. A is a uniform Banach algebra under the supnorm $\|$.$\| , A is also weakly regular.$ By [3], there exists a norm $|$.$| on C[0,1]$ such that $C[0,1]$ is an incomplete normed algebra. It is well known that $\|\| \leq.|$.$| . Let \mathrm{B}$ be the completion of $\mathrm{C}[0,1]$ under the norm $|$.$| . Consider$ $\Phi: \mathrm{A} \rightarrow \mathrm{B}, \Phi(\mathrm{f})=\mathrm{f}$, we have $(\operatorname{Im} \Phi)^{-}=\mathrm{B}$. If $\mathrm{B}$ is semisimple, then $\Phi$ is continuous, and consequently the norms $\|$.$\| and |$.$| are equivalent, a contradiction. Since \|\| \leq.||,. \Phi^{-1 /} \operatorname{Im} \Phi$ is continuous and so $\operatorname{Im} \Phi \cap \mathrm{R}\left((\operatorname{Im} \Phi)^{-}\right)=\{0\}$ by Theorem 3.1 .

Remark. The algebra A considered in the above example is also $\sigma^{*}$-compact-regular and $\operatorname{Im} \Phi \cap \operatorname{SR}\left((\operatorname{Im} \Phi)^{-}\right)=\{0\}$ but $\operatorname{SR}\left((\operatorname{Im} \Phi)^{-}\right) \neq\{0\}$

The following result is an application of Theorem 3.1.

Theorem 3.3. Let A be a functionally continuous normed algebra. Then the following assertions are equivalent:

(1) A is a uniform normed algebra.

(2) A has a largest closed, idempotent, absolutely convex, bounded subset.

Proof. (1) $=>$ (2): Let $\|$.$\| be a uniform norm defining the topology of A$. Let $B=\{x \in A,\|x\| \leq 1\}$, $\mathrm{B}$ is a closed, idempotent, absolutely convex, bounded subset of $\mathrm{A}$. Let $\mathrm{C}$ be an idempotent bounded subset of $A$. There exists $M>0$ such that $\|x\| \leq M$ for all $x \in C$. Let $x \in C,\|x\|=\left\|x^{2^{n}}\right\| 2^{2^{-n}} \leq M^{2^{-n}}$ for all $\mathrm{n} \geq 1$, then $\|\mathrm{x}\| \leq 1$ i.e. $\mathrm{x} \in \mathrm{B}$.

(2) $=>$ (1): Let B be a largest closed, idempotent, absolutely convex, bounded subset of A. By $\left[1\right.$, Proposition 2.15], we have $A=A(B)=\{t x, t \in \mathbb{C}$ and $x \in B\}$. Let $\|\cdot\|_{B}$ be the Minkowski functional of $\mathrm{B},\left(\mathrm{A},\|\cdot\|_{\mathrm{B}}\right)$ is a normed algebra. By [1, Proposition 2.15], $\beta=\|\cdot\|_{B}$ where $\beta$ is the radius of boundedness, then $\left(A,\|.\|_{B}\right)$ is a uniform algebra since $\beta\left(x^{2}\right)=\beta(x)^{2}$ for all $x \in A$. Let $A_{1}$ be the completion of $A$ under the original norm. It is clear that $\Phi:\left(A,\|.\|_{B}\right) \rightarrow A_{1}, \Phi(x)=x$, is continuous, and consequently $\left(\mathrm{A},\|.\|_{\mathrm{B}}\right)$ is functionally continuous. We now remark that we have proved the equivalence of (1), (2) and (3) in Theorem 3.1 without the condition that A is weakly regular. Using this remark, $\Phi^{-1} / \operatorname{Im} \Phi$ is continuous, then $\Phi$ is a homeomorphism (into), so $\mathrm{A}$ is a uniform normed algebra.

\section{REFERENCES}

[1] G.R.Allan, A spectral theory for locally convex algebras, Proc.London.Math.Soc. (3) 15 (1965), 399-421.

[2] A.Bedaa, S.J.Bhatt and M.Oudadess, On automatic continuity of homomorphisms, Proc.Amer.Math.Soc.Vol.128, No.4 (1999), 1039-1045.

[3] H.G.Dales, A discontinuous homomorphism from C(X), Amer.J.Math.101 (1979), 647-734. 
[4] H.V.Dedania, A seminorm with square property is automatically submultiplicative, Proc. Indian.Acad.Sci (Math.Sci), 108 (1998), 51-53.

[5] M.El Azhari, On some automatic continuity theorems, Bull.Belg.Math.Soc. 10 (2003), 627-634.

[6] A.Mallios, Topological algebras, Selected topics, North-Holland, 1986.

[7] E.A.Michael, Locally multiplicatively convex topological algebras, Mem.Amer.Math.Soc., No.11 (1952).

Ecole Normale Supérieure

Avenue Oued Akreuch

Takaddoum, BP 5118, Rabat

Morocco

E-mail: mohammed.elazhari@yahoo.fr 